\title{
Core-shell PVA/PVP-FeOOH nanofibers as potential visible light responsive drug delivery system
}

\author{
Anna Šutka ${ }^{1}$ \\ ${ }^{I}$ Biomaterials Research Laboratory, Institute of Inorganic Chemistry, Riga Technical University \\ e-mail: anna.sutka@rtu.lv
}

Core-shell electrospinning technique resolves the limitation in the traditional drug delivery methods allowing to load a higher number of therapeutic agents per unit mass of the polymer. Encapsulating the drug in the fibre core provides protection and prolonged drug release. The controlled drug release profile improves the effectiveness of drug therapy and can be tuned to meet the specific clinical application by modulating a variety of parameters, such as the drug to polymer ratio, fiber diameter, morphology, and porosity. The high surface area per unit volume and core-shell configuration of electrospun nanofiber mat has a potential to deliver drug nanocarriers with adsorbed drug molecules or therapeutic agents on surface.

In the present study mats of core-shell nanofibers were successfully formed by the coaxial electrospinning process, where a coaxial spinneret composed of an outer and inner needle is used. Further, composite nanofiber mats were developed with drug carrier introduced in fibre core as potential drug delivery system. The visible light responsive drug carriers were incorporated in fibre core to provide non-invasive drug release on demand. Light as an external stimulus for smart drug-delivery systems is advantageous for a number of reasons including its noninvasive nature, high spatial resolution and temporal control, and convenience and ease of use [1].

Here we demonstrate a system based on photothermal strategy where the adsorbed photon energy is dissipated as heat. The observed results show a potential for practical application as a visible light triggered drug release system. Reported phenomenon could open new possibilities to provide novel perspectives in wide range of applications for controlled drug delivery.

\section{Acknowledgements}

This work has been supported by the European Regional Development Fund within the Activity 1.1.1.2 "Post-doctoral Research Aid" of the Specific Aid Objective 1.1.1 "To increase the research and innovative capacity of scientific institutions of Latvia and the ability to attract external financing, investing in human resources and infrastructure" of the Operational Programme "Growth and Employment" (No. 1.1.1.2/VIAA/1/16/ 157).

\section{References}

1. Said, S. S.; Campbell, S.; Hoare, T. Chem Mater 2019, 31, 4971. 